\title{
"Interconnection" APP: Proposal of Interaction with a Virtual Agent, Animations and Augmented Reality an Easy Way to Learn the Usage of Sensors in Smart Cities
}

\author{
César Lozano Díaz ${ }^{1}$, Adriana Lorena Iñiguez Carrillo ${ }^{1}$, Rocio Maciel ${ }^{2}$, \\ Víctor M. Larios ${ }^{2}$, Emmanuel Aceves Martínez ${ }^{1}$, C. Alberto Ochoa ${ }^{3}$, \\ Edgar G. Cossio Franco ${ }^{4}$ \\ ${ }^{1}$ University of Guadalajara, Zapopan, Mexico \\ clozano@cucea.udg.mx, adriana.carrillo@cusur.udg.mx, \\ emmanuel.aceves@cucea.udg.mx \\ ${ }^{2}$ University of Guadalajara, Smart Cities Innovation Center, Zapopan, Mexico \\ rmaciel@cucea.udg.mx, vmlarios@cucea.udg.mx \\ ${ }^{3}$ Universidad Autónoma de Ciudad Juárez, Mexico \\ alberto.ochoa@uacj.mx \\ ${ }^{4}$ Instituto de Información Estadística y Geográfica de Jalisco, Mexico \\ kofrran@gmail.com
}

\begin{abstract}
Most of the technological resources used in a Smart Building environment are automated systems where sensors and their usability become a "black box" for their personnel (end user of these systems), giving in them a wrong impression about Who must be taking care of these resources and services? (These users think these services must be only provided and controlled by the own building), losing the culture of the correct usage of the regular services such as artificial climate, water services, electricity services, and the care of plants outside of these buildings. This paradigm of interaction between the user and the maintenance of resources of a smart building still needs improvement. For that reason, this current research is proposing the inter-connection of following resources: use of sensors, communication protocols, collections of MongoDB data, speech to text, text to speech and animations that represents feelings (sadness, happiness, worry) through the usage of Augmented Reality (AR) and IBM Watson conversation (Conversational Artificial Intelligence A. I.). The results show the architecture and favorable results about the viability to connect the services with natural interfaces. This approach helps and increments the user interaction with the places and objects that are around in the context of a Smart Building to understand the importance of the resources that are administrated and create a collective conscience through the correct usage of resources.
\end{abstract}

Keywords: smart building; interconnection; conversational agent; augmented reality.

\section{Introduction}

The implementation of technology based on simple interactions, intuitive and satisfactory improves the user experience. For that reason, researches have increased the development of new forms to interact with technology, and the world surrounds us to 
incorporate more natural forms of interaction for the user like the usage of speech, gestures, and visualization that enriches the visual experience like the Augmented Reality improving the quality of communication. These types of interfaces are called Natural User Interfaces (NUI). This interface aims to reduce the barrier between user and application, getting closer and closer to a way in which people interact with each other in their daily activities. The applications developed for the end user with NUI are capable of interacting with minimal training or null; the use of these principles of design appropriately, it will be easier the usage and execution of some tasks even for those users without experience. Along with the technological development, new tools have emerged and have revolutionized this interaction. For this research, the following tools have been identified.

The speech interaction has been used in diverse context, for instance, to active some instruction in immersed environments: education [1] or accesses to other services as a document, calendar or website. The Virtual and Augmented Reality have used in a wide variety of fields, such as entertainment [2], health [3], inclusion [4], education [5], manufacturing and logistics [6] and transportation [7], as well as the usage of sensors that allows monitoring Smart Buildings [8]. More specific in buildings the AR systems can help to read real-time measures such as temperature, oxygen, sound, movement, or energy consumption. Although, this last one requires friendly interfaces or previous training for the user to be able to understand this information.

Lastly, the growth of technologies for conversational agents with natural language is now used from simple request interfaces and response capability to full sentences, used in a whole range of scenarios, (customer support, air traffic control, or other situations). These technologies provide access to information in a faster way, and the learning curve is relatively short.

All these resources have been utilized in Smart cities environments in several ways, combining them or separately managing services in an automated way in the buildings [7]. However, in several cases, the usage of sensors administration and functionalities become a "black box" for the end users in these buildings. These systems at the building must provide the wrong impression to the society about the self-regulation and administration of the resources, and merely the fix of a sensor or the care of the resource must be managed by an IT expert only. This approach of smart buildings means that if desired to create collective conscience through the correct usage of resources such as water, electricity, the care of plants inside these buildings, it is necessary to ensure a direct and indirect interaction between the users and the improved services.

This paradigm of interaction between the user and the maintenance of resources of a smart building still needs improvement through an easy way to connect to the user with the services and interaction, similar to human or animal contact on a daily basis. It has not been well defined yet, therefore this research is proposing to implement the App develop with the possibility to interconnect the use of sensors, communication protocols, collections of MongoDB data, speech to text, text to speech and animations with AR that represents feelings (sadness, happiness, worry) adjusted to the measured parameters. The information used in sensors from the "interconnection" app through the Augmented Reality and a conversational system using an avatar form to improve these interactions. In this research, architecture and usability testing have exhibited favorable results connecting these technologies. That allows increasing the interaction and perception of the user about places and objects around in a context. For example, 
“Interconnection” APP: Proposal of Interaction with a Virtual Agent, Animations...

into a Smart Building, it is possible to provide the sensation that the plants and services are living beings that can interact in real time with the end user. Enabling the place for the users to ask questions and to receive responses with information of components condition to interact better with the building. The user interface was possible with the help of the conversational agent IBM Watson and the presentation in real time of the information that used in sensors.

\section{Background}

\subsection{Smart Building}

The vision of the cognitive building is to connect with the interactive design which operates proactively with the human activity inside them [10]. The exchange between the sensing and the systems occurs through the collection of data and the data process based on advance learning of technologies [11]; many of these buildings attempt to implement rules for different scenarios, for instance, the usage of energy [12]. The data is coming from sensors installed inside the building and systems that manage various functions defined to create or maintain an artificial environment. Another example will be a scene inside a school when a sensor informs the air quality inside the classroom. If the air is not good, the building will activate the ventilation, and the room will improve the comfort and the health of the individual. Also, the building will collect the consumption of energy to detect if the air conditioning system is working correctly [13].

\subsection{Augmented Reality}

AR allows users to see the real world, with virtual objects, superimpose or composed with another world [14]. Therefore, AR supplements the reality, instead of replacing it entirely. Ideally, it will seem to the user that the virtual and real objects coexist in the same space increasing the vision of the external world with the digital information. The real environment is aligned with the $3 \mathrm{D}$ space as a fusion of the content and context. That fusion is called the immerse multisensory environments and the applications of Augmented Reality provide opportunities for new electronic services for the Smart Cities.

In the context of mobile AR apps for smart cities, it can consider the so-called "video-see-through" apps: mobile applications that in real-time overlay information on top of the live video stream. The first and essential mobile AR apps used only the data coming from two sensors from the following devices: GPS receiver (for the location), and a digital compass (for the direction). [15]

Other categories of applications of the Augmented Reality (AR) are the montage, maintenance and fix of complex machines with instructions that are much easier to understand. Such information in AR replaces traditional instructions booklets with or text or images. AR can use 3D draws superposed to the real-time video, using a mobile device (Tablet of Smart Phone) showing step by step the tasks to develop and how to do them on its visual interface. 


\subsection{Speech Services}

In the past years two significative enhancements appeared in systems based on speech. Such systems, for example, are used in customer support and personal agents as well they integrate services into smart devices.

With the miniaturization of modern electronics, the implementation of Internet of things (IoT), the progress of artificial intelligence and the precision of the algorithms of speech recognition (Automatic Speech Recognition-ASR) and speech systems (Textto-Speech-TTS) it makes possible to use interfaces with the speech with natural language processing integrated into smart environments. Such interfaces are capable of acquiring and apply in knowledge over their population and their environment to adapt and compliance with their objectives with efficiency.

These interfaces must be sufficiently flexible to make use of a variety of devices, services, knowledge sources and user supplies. Also, with current processing capacities, speech interfaces are being able to manage simultaneously multiple tasks covering different aspects of communication, such as commands dialogues and controls, as well as the dialogues for recovering information [16].

\subsection{User Experience Assessment}

For the citizens in Smart Cities is essential to move freely and have personal or public devices allowing access to the services practically and straightforwardly. Looking to produce the experience of the user-friendly system systems to avoid a digital divide among citizens.

The user experience (UX) merged of the perception, the action, the motivation and the cognition in all the sense that defines the user interaction with a system $[17,18]$. The usability has been utilized to measure the user experience in the interactive systems. The ISO 9241-11 norm [19] defines the usability as "the extent to which a system, product or service can be used by specified users to achieve specified goals with effectiveness, efficiency, and satisfaction in a specified context of use". There are different methods and tools to measure the usability [20].

In general; three categories are considered: one at inspection methods, two for inquiry and three for testing. However, need to consider measuring the usability of the system with AR and the conventional systems. Also, it is necessary to consider the combination of real and virtual objects in scenarios and the necessity of real-time interaction.

\section{Proposed Approach}

\subsection{Interconnectivity and Model}

The growth of new technologies is allowing researchers to experiment with different methods of human-computer interaction. By giving the users an opportunity to understand the environment that surrounds us as an interactive and dynamic way in multimodal interfaces, creating exhaustive user studies to determinate how to implement this technology in relevant scenarios [21] showing its viability to support complex tasks 
interaction [22]. Proposing to define how an application needs the interaction before its development, this present research is based on the concept of Ambient and Active and Assisted Living (AAL) program, where the organizations need to share their common information coming from different sources and helping provide better assistance [23]. All the elements are interacting and are affected between them in such AAL [ 24]. An AAL detects and recognizes the actions, activities, and situations inside an environment, which use different sensors to recognize the activities and the comprehension of the behaviors [25].

A reference model is a RAModel [26], in which handles four dimensions to take in consideration: domain, application, infrastructure and transverse elements (Table1).

Table 1. Domain, application, and infrastructure.

\begin{tabular}{|c|c|c|}
\hline Domain & Application & Infrastructure \\
\hline $\begin{array}{l}\text { - Legislation, standard, } \\
\text { and regulation } \\
\text { - Quality attributes } \\
\text { - System compliance }\end{array}$ & $\begin{array}{l}\text { - Scope } \\
\text { - Functional } \\
\text { requirements } \\
\text { - Domain data } \\
\text { - Constraints } \\
\text { - Risks \& limitations } \\
\text { - Goals \& needs }\end{array}$ & $\begin{array}{l}\text { - Software elements } \\
\text { - Hardware elements } \\
\text { - Best practices \& } \\
\text { guidelines } \\
\text { - Arquitectural styles }\end{array}$ \\
\hline \multicolumn{3}{|c|}{ Crosscutting elements } \\
\hline - Decision & - Domain Terminology & - Communication \\
\hline
\end{tabular}

The main designs for the AAL systems adapted to the physical and cognitive capabilities, as well as the day to day activities, (ADL) of the user.

\section{$4 \quad$ Architecture of the System}

In this current research is being proposed the approaching with every user in the same manner they can access to the information obtained from sensors. The analysis of this information and modality of the system in Audio-Chabot mode through a conversational agent in the user's mobile device; this is done with the purpose of monitoring in real time the sensing results (understanding all the measured parameters), administrate and make them part of a preventive maintenance of the building services.

The document design uses the requirements of the IT managers of the Smart Cities Innovation Center at the University of Guadalajara CUCEA (not directly from the user, so a speculative interface to use is proposed, and improvements of the app expected in the future).

Therefore, the value of this proposal is in this app design "interconnectivity" in which is useful for the administrative services connected in the smart building (sensors that collect measures of temperature, humid, pollution, acoustics, air quality and light conditions), through IoT (Internet of Things) devices. All devices communicate in real time through the MQTT protocol sending information to the server, saving it in a nonrelational database MongoDB and taking the data to an application in AR to show the status of a sensor. In this way, the interconnection with the information used in the 
sensors with a speech to text services with a conversational agent could be "translated" by the agent linked to frequently asked questions. The user could ask (programmed in the Watson Conversation Service), getting responses with negligible value, supreme and ranks. The system can determine the optimal state of the service, errors, preventive actions or also a social interaction simulated (greeting, telling a joke, provide status in general of the room). All this to make the users part of preventive maintenance and they recognize the regulations of the system and create a collective conscience to the correct usage of the resources in a smart building.

In this way, the automation of these buildings it is not a simple action loop/ reaction, or else could be modified based on its most crucial human component. Like Rinaldi [27] proposing to take advantage of the digital capabilities with the power of cognitive computing. Cognitive systems, like IBM Watson TM, the system of questions and answers and derive conclusions of textual content.

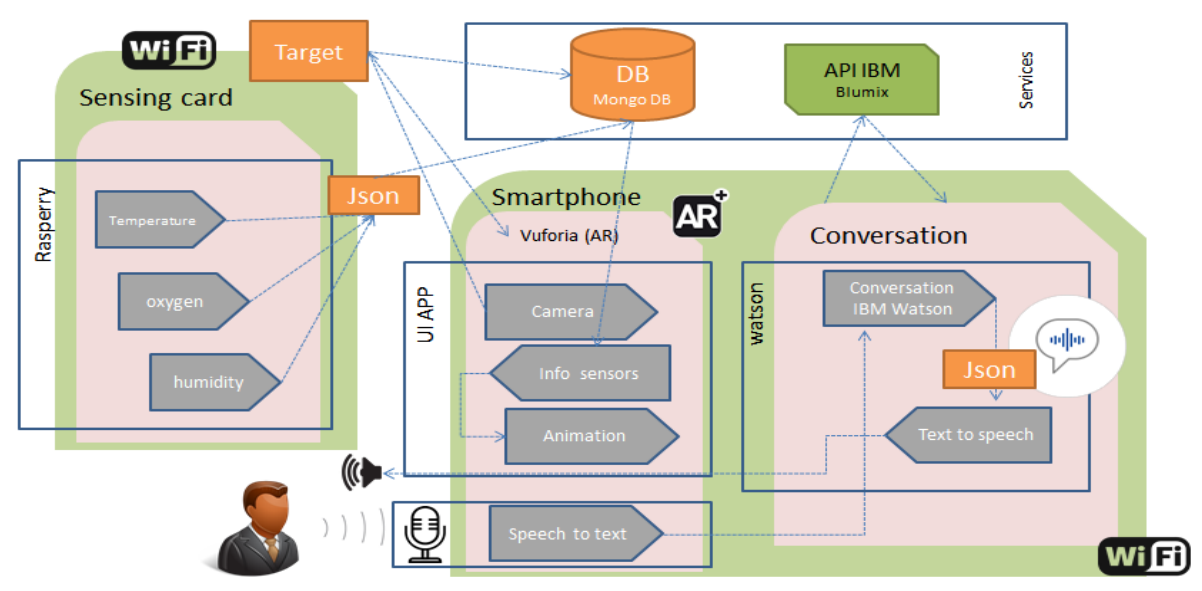

Fig. 1. The app interconnection between services.

Natural speech interaction is used, combined with other interactive modes to devise a multi-modal UI. Hence, researchers are experimenting with different modalities, often combining some of them into mixed interfaces and performing comprehensive user studies to determine their applicability in relevant scenarios and Multi-modal interfaces proved their viability in supporting complex interaction tasks. It is necessary to take into account that the research of a multi-modal interface to design need to have the following services: speech to text, text to speech, Augmented Reality, storing data in Mongo DB database and the service of IBM Blumix Watson conversation. This last service receives user inputs and processes them through the use of machine learning. With the analysis of natural language, it can assign defined intentions to entities. Reference Fig. 1.

The data transfer from the IoT devices is through the $\mathrm{WiFi}$, taking to the database MQTT the records. From Unity, the reception of the sensed data depends on each element of the building through Vuforia in Unity. This target also works as a reference for 
“Interconnection” APP: Proposal of Interaction with a Virtual Agent, Animations...

the conversational of Watson service. The data is stored to open the possibility to do analytics (Fig. 2).

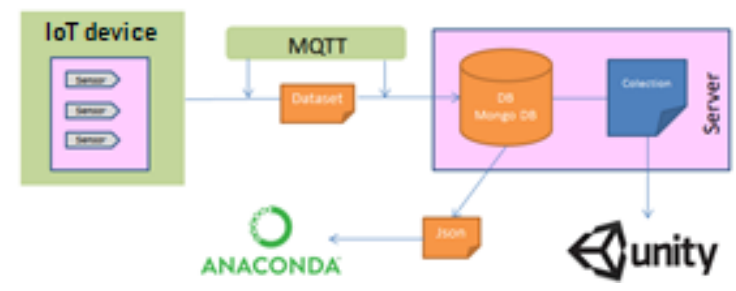

Fig. 2. Connection from Unity.

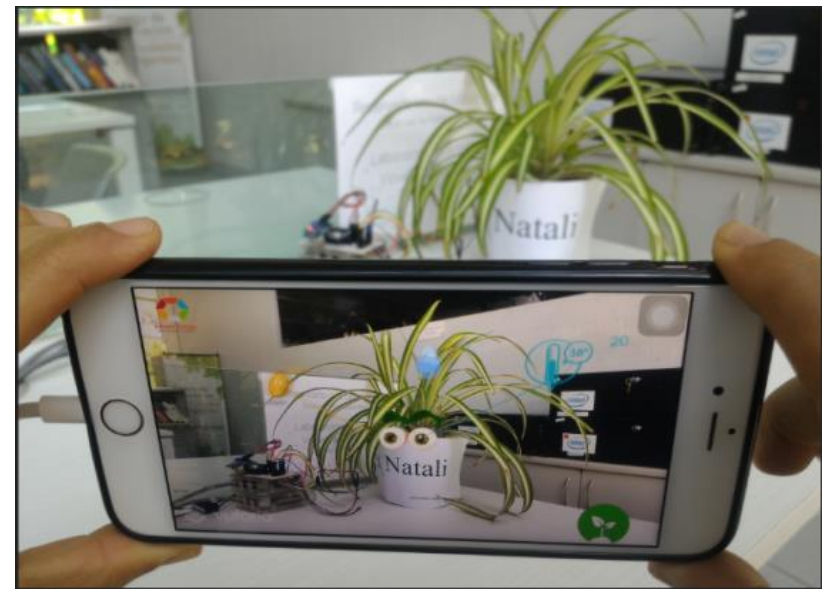

Fig. 3. UI utilized in a plant in the smart cities innovation center.

The data emerge to the UI through floating icons superimposed around the plant which each of the parameters measured (temperature, humid, luminous intensity). The interface also creates an AR with animated eyes and mouth. The animation depends on the "mood" of the plant, this base on the parameters measured, simulating sadness, happiness, worry. The system for speech to text is active in all moment; a button is added to start the conversation, in this case with the plant. Reference Fig. 3, the target is not a QR code else is tracking without marks base on Barroso [28] using the method of level two for AR, the user uses a microphone and speakers from their mobile phones to interact with the plant.

\section{Usability Test}

The assessed two evaluators, which they have experience in the AR concepts, conversational interfaces, and assessments in user experience. The study comprises an assessment of 14 volunteers $($ Men $=6$, Women $=8$ ) between professors and students of pregrade and postgraduate in the technology area, between ages from 18 to 54 . 
Individually assessment was done. First, a brief introduction of the app services to each one of the participants. Then, instructions were given to complete a series of activities (Reference Table 2). The participants were encouraged to ask questions and assistance was provided (in case of not completing with a specific task). When concluding the tasks, the participants could explore the application freely. In the end, asked the participants their feedback and they provided comments and suggestions to improve the user experience.

Table 2. Defined tasks.
(a) Open the application
(b) Greeting
(c) Knowing what type of plant is
(d) Knowing the plant's mood
(e) Discovering the ideal parameters for the plant
(f) Saying goodbye

The architecture assessment is based on the adaption of the questionnaire of the Subjective assessment of speech-system interfaces (SASSI) [25] and in the heuristics of AR [26]. The factors in the questionnaire SASSI were respected, and visibility factors were added to the AR system. Using a Likert scale of 5 points being ( $1=$ Disagreement until $5=$ Totally agree). With a total of 38 items and classified into seven factors, that analyzes the specific aspects of a system with speech recognition and AR graphs:

- A precise response of the system (RS): if the system is precise, reliable, predictable and with minor mistakes.

- Likeability (LK): if the system is useful, pleasant, friendly and easy to use.

- Cognitive Demand (DC): the level of concentration and ease use of the system.

- Annoyance (ML): if the interaction with the system is frustrating, repetitive or irritating.

- Habitability (HB): the certain that user has for the actions to be performed.

- Velocity(VZ): the system responds quickly or slowly.

- Visibility (VS): the images are useful, understandable and appropriate.

The results are shown of the questionnaire below: The participants showed a high level of likeability ( $M=4.05, \mathrm{SD}=0.99)$, with discreet feeling of frustration of the usage $(\mathrm{M}=2.37, \mathrm{SD}=0.39)$. Also, the users required minor effort when using the system (Cognitive Demand: $\mathrm{M}=3.46, \mathrm{SD}=0.46$ ). Although, the participants provided positive comments regarding how easy was the usage of the interaction of speech. It is necessary to increase the dialogues to use because in the RS (Precise response of the system: $M$ $=3.51, \mathrm{SD}=0.48$ ) shows signs that the users moderately agree of the precision with the system. That confirms a control variable without particular problems with the answers and its commands (Velocity: $\mathrm{M}=2.86, \mathrm{SD}=0.24$ ). The system behavior seemed to coincide with the conceptual model of the user (Skills: $\mathrm{M}=2.96, \mathrm{SD}=0.17$ ). Regarding the AR, the users mentioned the system is useful and appropriate (Visibility: $\mathrm{M}=4.68, \mathrm{SD}=0.43$ ). See Fig.4. 
In the assessment instrument validation and Cronbach's alpha is obtained with 0.807 , for each questionnaire with kurtosis centered in zero and no major of one.

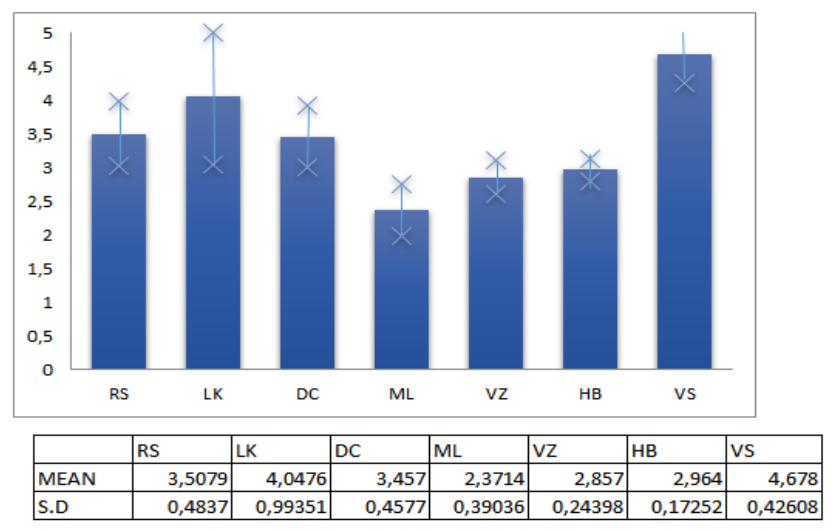

Fig. 4. Survey results.

The results of the assessment are showing essential results regarding the usage of AR and speech systems, as a potential benefit for improving the interaction in the systems using sensors in smart cities. The reactions of the participants were very positive, and they enjoyed the interaction with the system. Still, it is necessary to increase the dialogues, when doing a query there are different ways to do it, and as more vocabulary is in the systems, the system used in the plant could have a better understanding with the user.

\section{Discussion}

The AR applications in mobile devices frequently are handled through tactile controls and voice commands. However, if the possibility of monitoring the sensors in real time and the interaction with a conversational agent is added, it opens a possibility to access information in a faster and efficient way to the users to interact and know more about the results and their parameters. Also, combining the speech services with other forms of interaction to design multi-modal UI. Therefore, the researchers are experimenting with different methods, frequently combining some of them in mixed interfaces and the execution of exhaustive usability to determinate the application in relevant scenarios. The multi-modal interfaces have shown the viability in the tasks that support complex interactions [30].

As future research activities could be considered the interaction user talks combined with an AR management system integrated. Demonstrating an opportunity to take advantage of the IT usage as support of daily living activities. Also, it will interesting involve other groups of potential users that can take advantage of this interaction just using voice systems (such as blind people, or with any physical disability, like arthritis, motor paralysis). 
On the other hand, the proposal of interaction here presented is showing a possible way about the form in which it could work: AR manuals used for installation, assembling or fixing a product, with the possibility of asking Frequently Asked Questions (FAQ) to IT assistance in an immediate respond (Being this a conversational agent). Also, it is possible to implement assigned tasks for personal training, plant care and education as learning objects.

\section{Conclusion and Future Work}

The architecture and usability testing had provided favorable results about the viability to connect the AR speech recognition, here proposed. These technologies allow a significant increase with interaction and user perception about places and objects that are around in a context of a Smart Building giving the sensation that the plants and services are living beings when asking questions and receiving a response about their condition and activity of the building. Since the nature of the virtual component is not about a small component based on an image and audio but multimedia enriched with data of sensors in an animated way. Therefore, this represents a user advantage for the interaction and the easiest way to access the information of the people to be interviewed. New forms of information are provided, not just for the Smart buildings environments but also to deliver tutorials, training, educational resources, install manuals, without the necessity of using the augmented reality, with an essential impact for business and organizations innovation.

It is worth mentioning that the habitability and annoyance results done in usability test depends on some responses programmed in IBM Watson Conversation. In a future through the usability testing, an extensive content adjusted to the requirements will be programmed. Also, exists three areas of opportunity to continue developing this work inside the innovation ecosystems in smart cities:

1) The validation of evaluation instruments for the services of this type (since it does not count as this nature)

2) The implementation of this technology in an urban garden for the care of plants.

3) The incursion in the education with this type of technology to promote the care of the environment, through the care of plants and interaction with students.

\section{References}

1. Magal-Royo, T., Laborda, J.G.: Multimodal interactivity in foreign language testing. In: Multimodal Interaction with W3C Standards. pp. 351-365. Springer, Cham. (2017)

2. Magal-Royo, T., Laborda, J.G.: Multimodal interactivity in foreign language testing. In Multimodal Interaction with W3C Standards, pp. 351-365. Springer, Cham. (2017)

3. Matthew, H.G., et al.: Augmented Reality Technology Using Microsoft HoloLens in Anatomic Pathology. Archives of pathology \& laboratory medicine 142(5), 638-644 (2018)

4. Cerón, C., Archundia, E., Carcés, A., Beltrán, B., Migliolo, J.: Diseño de escenarios de aprendizaje con interfaces naturales y realidad aumentada para apoyar la inclusión de estudiantes con discapacidad auditiva en la educación media superior. Research in Computing Science 144, pp. 191-201 (2017) 
“Interconnection” APP: Proposal of Interaction with a Virtual Agent, Animations...

5. Ontiveros-Hernandez, N.J., Perez-Ramirez, M., Hernandez, Y.: Virtual Reality and Affective Computing for Improving Learning. Research in Computing Science 65, pp. 121-131 (2013)

6. Ingemar, K.et al.: Combining augmented reality and simulation-based optimization for decision support in manufacturing. In: Simulation Conference (WSC), 2017 Winter. IEEE, pp. 3988-3999 (2017)

7. Ghada, M., Essam; R., Khaled, H.: Augmented reality vehicle system: Left-turn maneuver study. Transportation research part C: emerging technologies 21(1), 1-16 (2012)

8. Conklin, J.A., Hammond, S.R.: Building integrated photovoltaic devices as smart sensors for intelligent building energy management systems. U.S. Patent No 9,772,260 (2017)

9. Hancke, G.P., et al.: The role of advanced sensing in smart cities. Sensors 13(1), 393$425(2012)$

10. Ciribini, A.L.C., et al.: Tracking users' behaviors through real-time information in BIMs: Workflow for interconnection in the Brescia Smart Campus Demonstrator. Procedia engineering (2017)

11. Rinaldi, S., Depari, A., Flammini, A., Vezzoli, A.: Integrating remote sensors in a smartphone: The project "sensors for ANDROID in embedded systems". In: 2016 IEEE Sensors Applications Symposium (SAS), Catania, Italy, April 20-22, pp. 468-473, ISBN 978-1- 4799-7249-4, DOI 10.1109/SAS.2016.7479892 (2016)

12. Sianaki, O.A., Hussain, O., Dillon, T., Tabesh, A.R.: Intelligent Decision Support System for Including Consumers' Preferences in Residential Energy Consumption in Smart Grid. In: Proc. of International Conference on Computational Intelligence, Modelling and Simulation (CIMSiM), 28-30 Sept., Bali, pp. 154-159 (2010)

13. Rinaldi, S., et al.: Bi-directional interactions between users and cognitive buildings by means of a smartphone app. In: Smart Cities Conference (ISC2), 2016 IEEE International. IEEE, pp. 1-6. (2016)

14. Azuma, R.T.: A survey of augmented reality. Presence: Teleoperators \& Virtual Environments 6(4), 355-385 (1997)

15. Graham, M., Zook, M., Boulton, A.: Augmented reality in urban places: contested content and the duplicity of code. Transactions of the Institute of British Geographers 38(3), 464479 (2013)

16. De la Cruz-Martinez, G., Eslava-Cervantes, A.L., Castañeda-Martínez, R.: Diseño de la Experiencia del Usuario para Espacios Interactivos de Aprendizaje no Formal. Research in Computing Science 89, pp. 53-62 (2015)

17. Heinroth, T., Minker, W.: "Next Generation Intelligent Environments: Ambient Adaptative Systems" Springer Press (2011)

18. Diamantaki, K.: Evaluating the user experience of a mobile user in a smart city context. January https://doi.org/10.1504/IJIEI.2015.069902 (2015)

19. International Standards Organization: ISO 9241-11:2186(en), Ergonomics of human-system interaction - Part 11: Usability: Definitions and concepts. https://www.iso.org/obp/ui/\#iso:std:iso:9241:-11:ed-2:v1:en

20. Mahrin, M.N., Strooper, P., Carrington, D.: Selecting usability assessment methods for software process descriptions. In: Proceedings of the Asia-Pacific Software Engineering Conference, pp. 523-529, Washington, DC, USA (2009)

21. Crowell, C.: Analysis of Interaction Design and Assessment Methods in Full-Body Interaction for Special Needs. In: 23rd International Conference on Intelligent User Interfaces. ACM, pp. 673-674 (2018)

22. Gutiérrez, M., Thalmann, D., Vexo, F.: Semantic virtual environments with adaptive multimodal interfaces. In: Multimedia Modelling Conference, 2005. MMM 2005. Proceedings of the 11th International. IEEE, pp. 277-283 (2005)

23. Cavallo, F., Aquilano, M., Arvati, M.: An ambient assisted living approach in designing domiciliary services combined with innovative technologies for patients with Alzheimer's 
César Lozano Díaz, Adriana Lorena Iñiguez Carrillo, Rocio Maciel, Víctor M. Larios, et al.

disease: a case study. American Journal of Alzheimer's Disease \& Other Dementias 30(1), 69-77 (2015)

24. Feder-Levy, E., Blumenfeld-Liebertal, E., Portugali, J.: The well-informed city: A decentralized, bottom-up model for a smart city service using information and self-organization. In: Smart Cities Conference (ISC2), 2016 IEEE International, pp. 1-4 (2016)

25. El murabet, A., et al.: Ambient Assisted living system's models and architectures: A survey of the state of the art. Journal of King Saud University - Computer and Information Sciences, https://doi.org/10.1016/j.jksuci.(2018)

26. Nakagawa, E.Y., Oquendo, F., Becker, M.: RAModel: A Reference Model for reference Architectures. https://doi.org/10.1109/WICSA-ECSA.212.4, pp. 297-301 (2012)

27. Rinaldi, S., et al.: Bi-directional interactions between users and cognitive buildings by means of smartphone app. In: Smart Cities Conference (ISC2), 2016 IEEE International, pp. 1-6 (2016)

28. Ranjan, R., Gan, W.S.: Natural listening over headphones in augmented reality using adaptive filtering techniques. IEEE/ACM Transactions on Audio, Speech, and Language Processing 23(11), 1988-2002 (2015)

29. Barroso Osuna, J.M., Cabero Almenara, J., García-Jiménez, F., Calle-Cardoso, F.M., Gallego-Pérez, Ó., Casado-Parada, I.: Diseño, producción, evaluación y utilización educativa de la realidad aumentada. (2017)

30. Gerard, S.N., Megerian, M.G.: Extracting semantic relationships from table structures in electronic documents. U.S. Patent No. 8,914,419. vol. 180, pp. 1484-1494 (2014) 